\title{
EL PROGRAMA INTERNO PARA EL CUIDADO Y USO DE LOS ANIMALES DE LABORATORIO EN LAS INSTITUCIONES BIOMÉDICAS DOCENTES, DE INVESTIGACIÓN CIENTÍFICA E INDUSTRIA FARMACÉUTICA
}

\author{
Jorge Fernández Hernández* e Yvonne Michelle Heuze de Icaza**
}

\begin{abstract}
Resumen: Este artículo tiene por objetivo analizar la ética en el cuidado y uso de los biomodelos experimentales denominados "animales de laboratorio".

No pretende establecer un modelo, sino describir los aspectos que cada institución que produce, mantiene y utiliza animales de experimentación, para fines académicos y/o productivos, debe tener en cuenta en vistas a un programa institucional de cuidado y uso con los más altos estándares científicos, técnicos y éticos.

De igual forma, analiza la percepción de este problema en la sociedad y la preocupación acerca del uso de los animales de laboratorio en la investigación científica.
\end{abstract}

Palabras clave: ética, animales de laboratorio, programa de cuidado y uso

\section{THE INNER PROGRAM FOR THE CARE AND USE OF LABORATORY ANIMALS IN BIOMEDICAL, RESEARCH AND PHARMACEUTICAL INSTITUTIONS}

\begin{abstract}
This paper has as goal to analyze the ethics of the care and use of experimental biomodels known as "laboratory animals".

It does not pretend to establish a unique model, but to describe the items to be considered by institutions which produce, maintain and use laboratory animals for academic and/or productive purposes, in order to establish an institutional program for the care and use of animals with the highest scientist, technical and ethical standards.

In the same way, it reviews the public perception on the topic and the ethical concerns about the use of laboratory animals in scientific research.
\end{abstract}

Key words: ethics, laboratory animals, animal care and use program

\section{PROGRAMA INTERNO PARA O CUIDADO E USO DE ANIMAIS DE LABORATÓRIO NAS INSTITUIÇÕES BIOMÉDICAS UNIVERSITÁRIAS E DE PESQUISA CIENTÍFICA E A INDÚSTRIA FARMACÊUTICA}

Resumo: Este artigo tem por objetivo analisar a ética do cuidado e o uso dos biomodelos de pesquisa denominados “animais de laboratório". Longe de pretender estabelecer um modelo, visa descrever os aspectos que cada instituição deve levar em conta ao produzir, manter e utilizar animais de experimentação, para fins acadêmicos e/ou produtivos. Esta instituição deve ter um programa institucional de cuidado e uso com os mais altos standars científicos, técnicos e éticos. Igualmente, analisa a percepção deste problema na sociedade e a preocupação sobre o uso dos animais de laboratório na investigação científica.

Palavras chave: ética, animais de laboratório, programa de cuidado e uso

* Especialista en Gnotobiología. Jefe de la Unidad de Producción y Experimentación de Animales de Laboratorio del Centro de Investigación y Estudios Avanzados del IPN (Cinvestav), México

Correspondencia: jofernan@cinvestav.mx

** Licenciada en Medicina Veterinaria y Zootecnia. Coordinadora de la Unidad de Producción y Experimentación de Animales de Laboratorio - Bioterio de la Universidad Autónoma Metropolitana, México

Correspondencia: ymheuze@correo.xoc.uam.mx 


\section{Introducción}

El uso de los animales de laboratorio en el mundo de hoy en los inicios del siglo XXI -dadas las implicaciones éticas y productivas, de investigación científica, académicas y financieras vinculadas con la salud del hombre y de los propios animales- requiere, por las características del animal de laboratorio en su condición de "reactivo vivo", de un manejo y un uso que impliquen la garantía total de aplicación de estándares internacionales, resultados de trabajo del más alto nivel y, por consiguiente, de alta confiabilidad.

En un mundo globalizado, en el cual la palabra "competitividad" adquiere un significado multinacional, es imprescindible adquirir hábitos y establecer mecanismos que den certeza de la calidad del proceso científico productivo y de sus resultados.

Los resultados en la investigación científica y la docencia universitaria en las disciplinas biomédicas -en cualquier parte del mundo, sin importar su carácter público o privado- y los resultados económicos en la multimillonaria industria médico farmacéutica internacional están relacionados, en mayor o menor grado, con la calidad de los animales de laboratorio y el entorno ético que en su cuidado y uso exige hoy la opinión pública.

Una de las vías para lograr resultados productivos, económicos y éticos aceptados por la mayoría de los interlocutores en estos campos de acción, ya sean científicos, técnicos, corporativos y por la opinión pública, es la introducción de principios de trabajo que conlleven la aplicación de estándares internacionales, bajo un esquema de procedimientos operacionales de trabajo, de subprogramas y de su verificación, y de control institucional, local y federal por medio de los órganos o entidades competentes.
El centro de atención y de ejecución de estas actividades es, sin duda, la institución en sí, y en ella se debe reflejar la necesidad de alcanzar estos estándares y de aplicar las medidas necesarias para que todo lo relacionado con el cuidado y uso de los animales se encuentre "institucionalizado". El Comité de Ética o Comité Institucional (o Interno) para el Cuidado y Uso de Animales de Laboratorio debe tener la prerrogativa de discutir y proponer a las autoridades institucionales las políticas relacionadas con el tema y, una vez aprobadas, establecerlas y vigilar su cumplimiento.

\section{Composición de un Programa Interno para el Cuidado y Uso de Animales de Laboratorio (PICUAL)}

Todo programa que se establezca de forma institucional, relacionado con el cuidado y uso de los animales de laboratorio, deberá contar con cuatro elementos esenciales que interactúen entre sí (figura 1).

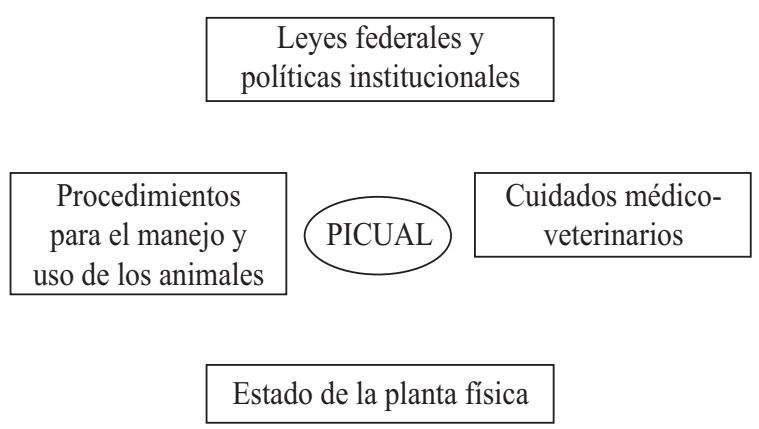

Figura 1.- Elementos esenciales de un PICUAL

Estos elementos representan cuatro grupos, en los cuales se conjugan las actividades de carácter científico, las tareas de índole técnica, las responsabilidades personales e institucionales y las características y condiciones físicas de las instalaciones en las que se realiza el trabajo con animales de laboratorio.

La conjunción práctica de estas actividades y su interacción generan un complejo número de tareas y responsabilidades en las que 
intervienen los participantes claves en la formación del PICUAL (figura 2), que deben ser orientadas y supervisadas por el Comité Institucional para el Cuidado y Uso de los Animales de Laboratorio (CICUAL), el que, a su vez, debe mantener informado a la máxima dirección institucional del estado de cumplimiento del mismo.

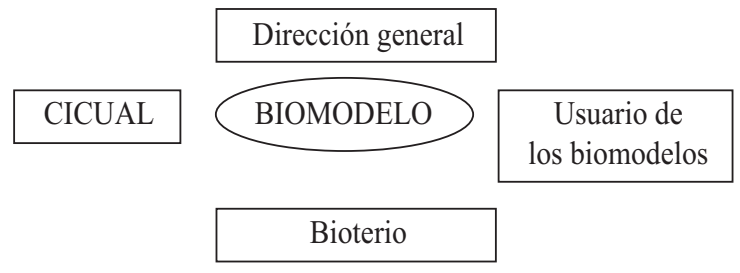

Figura 2. Participantes claves en el Programa Institucional para el Cuidado y Uso de los Animales de Laboratorio (PICUAL).

\section{Factores que influyen directamente en la calidad de los animales de laboratorio}

Todo lo anterior interviene, en mayor o menor grado, en la calidad final del animal como sujeto experimental y, por consiguiente, en el resultado parcial o total de un procedimiento experimental como parte de un protocolo de investigación científica.

Estos factores, representados en general en la figura 3, deben ser considerados dentro del PICUAL como parte de las políticas institucionales y desarrollados unos como procedimientos operacionales de trabajo, otros como subprogramas internos. Por ejemplo: el de capacitación de técnicos, profesionales, miembros del CICUAL, estudiantes; el de bioseguridad o seguridad ocupacional, etc.

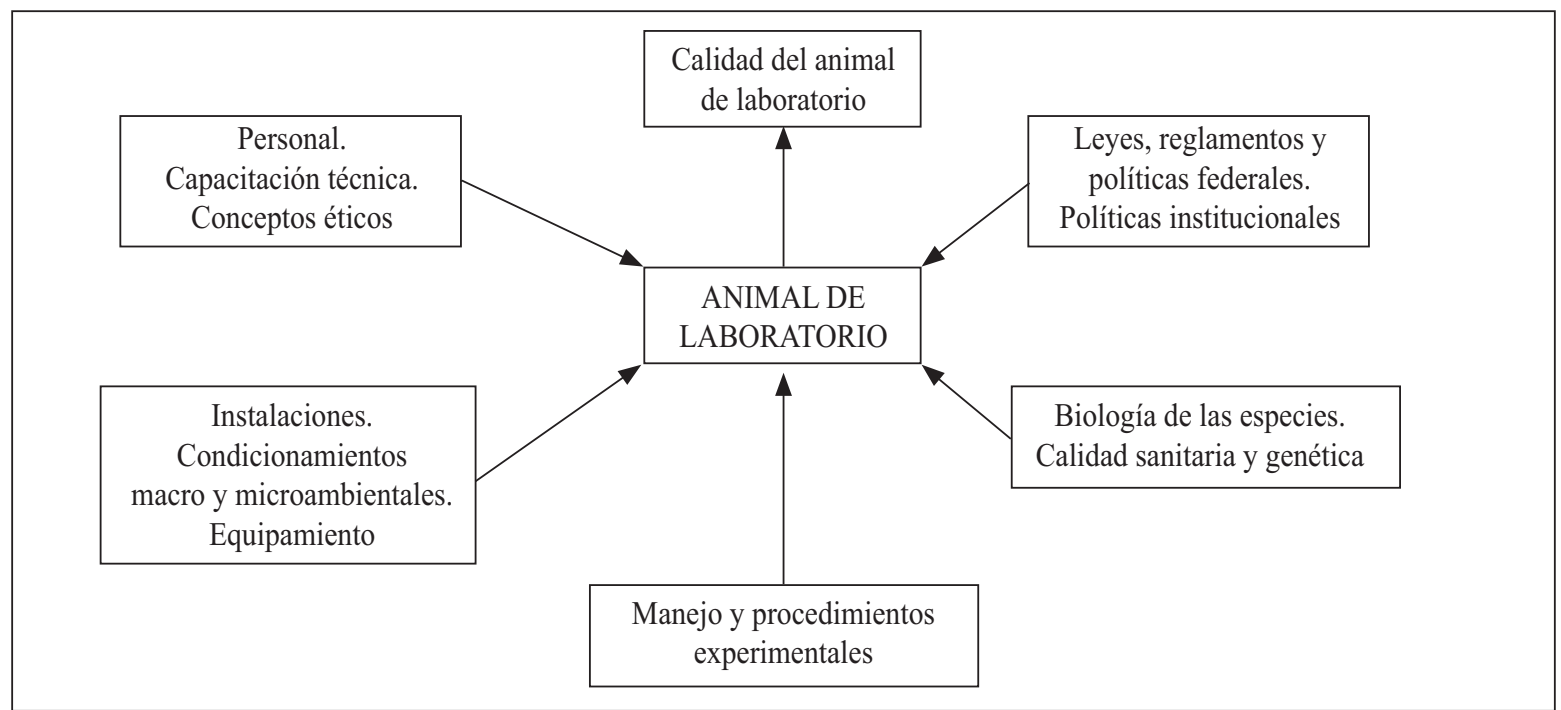

Figura 3.- Factores que influyen directamente en la calidad de los animales de laboratorio.

Composición del Programa Interno para el Cuidado y Uso de los Animales de Laboratorio

\section{Subprograma de Responsabilidad Institucional}

Este elemento adquiere una importancia estratégica, pues es el fundamento del programa, en el cual se establecen las políticas internas para el cuidado, el trato y el uso humanitario de los animales, la obtención de recursos necesarios para el mantenimiento de las instalaciones, la adquisición del equipo, las nuevas inversiones, etc. También, establece las bases del programa que estarán en correspondencia con las normas, reglamentos y leyes nacionales que rigen el cuidado y uso de animales, pudiéndose considerar también otros documentos como guías y similares existentes en otras regiones del mundo. 
La responsabilidad de la creación y del trabajo del CICUAL recae en la máxima autoridad de la institución, y la ejecución y constatación del programa es responsabilidad del mismo CICUAL. Debe establecerse el reglamento para su creación y funcionamiento así como los deberes y responsabilidades que adquiere con la institución y con los usuarios de los animales. Entre las principales tareas del CICUAL se destacan dos:

- Revisión de los protocolos para el cuidado y uso de los animales de laboratorio, documento que todo el que utilice animales estará en la obligación de redactar y de esperar por dictamen del CICUAL.

- Inspecciones -con la periodicidad que se considere necesaria internamente o aquella establecida por ley-a las actividades científicas que usen animales y a las instalaciones donde se alojan y/o utilizan.

El CICUAL debe reunirse regularmente para tratar todo asunto relacionado con el trabajo con animales y la calidad de los mismos, para la evaluación de los protocolos, para el cuidado y uso de animales y para la atención de los problemas que se presenten, entre otros temas a tratar.

Otro aspecto dentro de las responsabilidades institucionales son las regulaciones relacionadas con los protocolos que implican restricción física del animal, múltiples intervenciones quirúrgicas en un mismo biomodelo y restricciones de agua y/o alimento.

La atención médico veterinaria, como política para garantizar la atención correcta a los animales y la preparación profesional idónea, y la capacitación técnica y los requisitos para el conocimiento teórico y práctico del personal que trabaja con animales son otros de los aspectos esenciales que debe supervisar.
Es fundamental considerar, dentro de las responsabilidades institucionales, los temas de salud ocupacional y la seguridad del personal en todas las instituciones pero, sobre todo, en aquellas donde se trabajan con organismos patógenos.

\section{Subprograma de Manejo de los Animales de Laboratorio}

Este tema es vital y da cuenta de una relación directa entre las técnicas y condiciones de manejo de los animales y el trato ético hacia los mismos.

Un adecuado ambiente (macro y micro) y un manejo profesional requieren de la consideración de varios factores:

- Las características de las especies con las que se trabajará.

- El diseño y construcción del alojamiento primario y secundario.

- El enriquecimiento ambiental.

- Los objetivos de la institución (experimentación científica, pruebas de constatación y/o educación media o superior).

- Los procedimientos experimentales.

- El uso de microorganismos patógenos, sustancias químicas, tóxicas, radioactivas, etc.

A continuación se relacionan los aspectos del este subprograma que es necesario tener presente en la formación del PICUAL:

a. Macro y microambiente.

b. Alojamiento (encierro primario y secundario e instalaciones al aire libre).

c. Recomendaciones de espacio.

d. Variables ambientales (temperatura, humedad, ventilación, ruido, iluminación, etc.). 
e. Atención directa (alimentación, agua, encamado, limpieza, etc.).

f. Emergencias.

g. Identificación de los animales y registro clínico.

\section{Subprograma de Atención Médico Veterinaria}

Se deben considerar los aspectos que incluyan:

- Vigilancia, diagnóstico, tratamiento y control de enfermedades.

- Manejo de enfermedades asociadas al protocolo de uso de animales.

- Anestesia, analgesia y eutanasia. Vigilancia del dolor.

- Cirugía con y sin recuperación.

- Bienestar animal.

- Cuarentena y adaptación.

- Registro y control de incidencias.

\section{Subprograma de Instalaciones Físicas}

Es necesario prestarle especial atención a las condiciones físicas de las instalaciones, su cuidado y mantenimiento. Es importante el diseño arquitectónico cuando se inician los estudios para una nueva instalación: considerar las áreas funcionales, las guías para la construcción y las especificaciones precisas para aquellos casos de áreas especiales como quirófanos, de bioseguridad, cuartos de procedimientos experimentales, etc. No se deben olvidar las condiciones que deben reunir los espacios donde serán colocados los equipos de considerable tamaño, como las autoclaves, lavadoras, SVAAC, entre otros. Es preciso considerar:
- Las necesidades experimentales de los usuarios referentes a las áreas que serán utilizadas para realizar sus trabajos con los biomodelos.

- Las características y condiciones que exigen las especies de animales que serán alojadas en cada una de las áreas de toda la instalación física.

\section{La investigación científica con animales de laboratorio y su relación con la bioética y la sociedad}

Moral, ética, derechos de los animales, trato humanitario, procedimientos éticos, dolor innecesario, reemplazo, reducción, refinamiento y responsabilidad son conceptos de los que se habla con pasión cuando el tema surge en un análisis de caso o en la evaluación de un protocolo para el uso de animales, entre otras situaciones.

El tópico del uso o no de animales en la investigación científica, como biomodelos experimentales, ha sido discutido por muchos años y como es imposible tratar este tema con la profundidad que el mismo exige, por su importancia y por su implicación social, sólo mencionaremos algunos elementos importantes de considerar.

Todos los países que promueven y tienen leyes que protegen a los animales, de carácter local o federal, contemplan de forma implícita en su contenido y esencia los elementos éticos para el trato a los animales que hemos señalado.

Por supuesto que una cosa es lo legislado por los parlamentos y la obligación de su verificación por parte de las autoridades administrativas responsables, y otra es el comportamiento práctico del individuo para con los animales en su entorno social. 
Cuando existe constatación del cumplimiento de las leyes por parte de las autoridades responsables el comportamiento de un individuo se rige por ellas y, entonces, el sistema funciona bien en general. Cuando esta relación tiene sus fallas y las autoridades no cumplen con su papel fiscalizador las leyes no sirven de nada y el individuo hace lo que su ética personal (o falta de ética) le dicta.

La sociedad civil - en su carácter de institución académica, de educación o de laboratorio de pruebas, de sociedad protectora de animales o de individuo preocupado por la protección animal y por la aplicación de los esquemas de carácter ético para con los animales- es la encargada de hacer cumplir la ley.

En este esquema social entre los que usan animales y los que quieren protegerlos hay, por lo general, dos formas de comportamiento:

- aquella que manifiesta posiciones extremistas y, por consiguiente, encontradas y con casi ningún punto de coincidencia entre los actores, $\mathrm{y}$

- aquella que manifiesta y promueve posiciones coincidentes respecto al cuidado y uso de carácter ético de los animales.

\section{La sociedad y la experimentación científica con animales}

Por lo general, la sociedad-cuando existe una adecuada divulgación del papel de la ciencia, en conjunción con el uso de animales bajo principios éticos que cumplan con el objetivo de encontrar respuestas y tratamientos eficaces a las enfermedades que afectan la calidad de la vida humana y la de los propios animales- acepta el papel que juega el animal de laboratorio como biomodelo experimental.
Una encuesta de Associated Press (AP) en Estados Unidos, realizada en 1995 (Management of Laboratory Animal Care and Use Programs, CRC Press 2002, página 102), dio como resultado lo siguiente:

- $67 \%$ de la población creía que los animales tenían el mismo derecho de vivir sin sufrir que los humanos.

- $59 \%$ sintió que es incorrecto usar para vestir las pieles de los animales.

- $67 \%$ se oponía al uso de animales en las pruebas de cosméticos.

- $70 \%$ consideraba correcto el uso de animales con fines de investigación científica en el tratamiento de enfermedades.

- $98 \%$ del total de personas encuestadas consumía carne de origen animal dentro de su dieta habitual.

La aprobación social para el uso de animales en la investigación científica biomédica es evidente, mas no se encuentra exenta de calificación y este uso es considerado adecuado siempre y cuando:

- Los animales sean tratados humanamente.

- No estén sujetos a procedimientos experimentales que causen dolor sin el uso de analgésicos y anestésicos.

- Los estudios reflejen información útil para el descubrimiento y los avances de la ciencia médica y veterinaria.

Sondeos más recientes, como el realizado por Market and Opinion Research International (MORI) el año 2005, a solicitud de Coalition for Medical Progress (CMP) en Gran Bretaña, demostró que: 
- $\quad 75 \%$ de la población de este país acepta la experimentación animal siempre que sea con propósitos de investigación biomédica.

- $76 \%$ de los encuestados acepta la experimentación animal mientras no conlleve al sufrimiento innecesario de los animales.

- $72 \%$ de los adultos está de acuerdo con la experimentación animal para todo tipo de investigación biomédica donde no existan alternativas para el uso animal.

- 53\% acepta la investigación con animales sólo para el tratamiento de enfermedades que ponen en peligro la vida.

- $89 \%$ de todos los entrevistados están de acuerdo con una o más de las cuatro respuestas anteriores.

\section{La importancia del animal de laboratorio}

Los animales han ocupado un lugar predominante en los descubrimientos médicos que le han proporcionado a más de 70 científicos de todo el mundo el Premio Nobel en Medicina y Fisiología, desde 1901 hasta la fecha (American Association for Laboratory Animal Science).

A manera de ejemplo, entre estos importantes descubrimientos mencionaremos los siguientes, sin restarle méritos e importancia a los no mencionados:

- El descubrimiento del ciclo de la malaria por Ross, en 1902, utilizando palomas como biomodelo experimental.

- Theiler, en 1951, desarrolló la vacuna contra la fiebre amarilla utilizando primates y ratones.

- De Duve, Palade y Claude descubrieron, en 1974, la organización funcional y estructural de la célula utilizando pollos, cobayos y ratas.

- Murria y Tomas, en 1990, utilizando perros de laboratorio, mejoraron la aplicación de técnicas para los trasplantes de órganos.

- Lauterbur y Mansfield descubrieron, en 2003, aspectos esenciales en el uso de la Resonancia Magnética (MRI) utilizando almejas, ranas, ratas, ratones, perros, cerdos y primates no humanos.

Es determinante lograr un equilibrio entre el eficiente uso de los animales, desde el punto de vista experimental y sus resultados, y los conceptos éticos que debemos aplicar durante la experimentación, la docencia y el control de medicamentos.

\section{Bibliografía}

Comité Consultivo Nacional de Normalización de Protección Pecuaria, Dirección General de Salud Animal, NOM-062-ZOO-1999. Especificaciones Técnicas para la Producción, Cuidado y Uso de los Animales de Laboratorio; 1991.

Hau J, Van Hoosier G. Handbook of Laboratory Animal Science. Vol. I, Essential Principles and Practices. Boca Raton: CRC Press; 2003.

Institute of Laboratory Animal Resources, Commission on Life Sciences, National Research Council. Guide for the Care and Use of Laboratory Animals. Washington DC: National Academic Press; 1996. 
Programa interno para el cuidado y uso de los animales de laboratorio - J. Fernández e Y. Heuze de Icaza

Programa Institucional para el Cuidado y Uso de los Animales de Laboratorio del Centro de Investigación y Estudios Avanzados del IPN. PICUAL-Cinvestav; 2002.

Suckow MA, Douglas FA, Weichbrod RH. Management of Laboratory Animal Care and Use Programs. Boca Raton: CRC Press; 2002.

Recibido el 15 de diciembre de 2006.

Aceptado el 12 de febrero de 2007. 